Acta Crystallographica Section E

Structure Reports

Online

ISSN 1600-5368

\section{Bis(4-ethoxyphenyl) sulfoxide}

\section{Kang Meng, Cheng Wu, Jing Cao, Ping Ma and Lihong Liu*}

Pharmacy Department of the Second Artillery General Hospital, Beijing 100088, People's Republic of China

Correspondence e-mail: lihongliu2011@yahoo.cn

Received 25 March 2011; accepted 8 April 2011

Key indicators: single-crystal X-ray study; $T=113 \mathrm{~K}$; mean $\sigma(\mathrm{C}-\mathrm{C})=0.002 \AA$; disorder in main residue; $R$ factor $=0.034 ; w R$ factor $=0.095$; data-to-parameter ratio $=18.1$

In the title compound, $\mathrm{C}_{16} \mathrm{H}_{18} \mathrm{O}_{3} \mathrm{~S}$, the dihedral angle between the benzene rings is $82.7(2)^{\circ}$. The $\mathrm{O}$ atom of the sulfoxide group is disordered over two orientations with refined occupancy factors of $0.563(3): 0.437$ (3). In the crystal, molecules are linked by intermolecular $\mathrm{C}-\mathrm{H} \cdots \mathrm{O}$ hydrogen bonds, forming chains along the $b$ axis.

\section{Related literature}

For background to Friedel-Crafts acylation, see: Edward \& Sibelle (1963); DeHaan et al. (1979); Fillion \& Fishlock (2005); Nishimoto et al. (2008). For the structures of related arylsulfoxides, see: Casarini et al. (2004); Noland \& Kedrowski (2000).

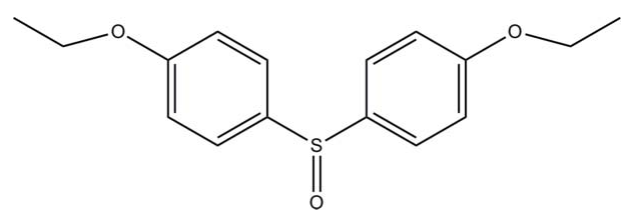

\section{Experimental}

Crystal data

$\mathrm{C}_{16} \mathrm{H}_{18} \mathrm{O}_{3} \mathrm{~S}$

$M_{r}=290.36$

Triclinic, $P \overline{1}$

$$
\begin{aligned}
& \alpha=64.71(3)^{\circ} \\
& \beta=83.78(3)^{\circ} \\
& \gamma=82.88(3)^{\circ} \\
& V=738.4(3) \AA^{3} \\
& Z=2
\end{aligned}
$$

Data collection

Rigaku Saturn CCD area-detector diffractometer

Absorption correction: multi-scan (CrystalClear; Rigaku, 2007)

$T_{\min }=0.957, T_{\max }=0.974$

\section{Refinement}

$R\left[F^{2}>2 \sigma\left(F^{2}\right)\right]=0.034$

$w R\left(F^{2}\right)=0.095$

$S=1.06$

3450 reflections
Mo $K \alpha$ radiation

$\mu=0.22 \mathrm{~mm}^{-1}$

$T=113 \mathrm{~K}$

$0.20 \times 0.16 \times 0.12 \mathrm{~mm}$

6624 measured reflections 3450 independent reflections 2590 reflections with $I>2 \sigma(I)$ $R_{\text {int }}=0.022$

Table 1

Hydrogen-bond geometry $\left(\AA{ }^{\circ}\right)$.

\begin{tabular}{lllll}
\hline$D-\mathrm{H} \cdots A$ & $D-\mathrm{H}$ & $\mathrm{H} \cdots A$ & $D \cdots A$ & $D-\mathrm{H} \cdots A$ \\
\hline $\mathrm{C} 11-\mathrm{H} 11 \cdots \mathrm{O}^{\mathrm{i}}$ & 0.93 & 2.51 & $3.3013(18)$ & 143 \\
\hline
\end{tabular}

Symmetry code: (i) $x, y+1, z$.

Data collection: CrystalClear (Rigaku, 2007); cell refinement: CrystalClear; data reduction: CrystalClear; program(s) used to solve structure: SHELXS97 (Sheldrick, 2008); program(s) used to refine structure: SHELXL97 (Sheldrick, 2008); molecular graphics: SHELXTL (Sheldrick, 2008); software used to prepare material for publication: SHELXTL.

Supplementary data and figures for this paper are available from the IUCr electronic archives (Reference: RZ2577).

\section{References}

Casarini, D., Lunazzi, L., Mazzanti, A., Mercandelli, P. \& Sironi, A. (2004). J. Org. Chem. 69, 3574-3577.

DeHaan, F. P., Covey, W. D., Delker, G. L., Baker, N. J., Feigon, J. F., Miller, K. D. \& Stelter, E. D. (1979). J. Am. Chem. Soc. 101, 1336-1337.

Edward, J. W. R. \& Sibelle, E. C. (1963). J. Org. Chem. 28, 674-676.

Fillion, E. \& Fishlock, D. (2005). J. Am. Chem. Soc. 127, 13144-13145.

Nishimoto, Y., Babu, S. A., Yasuda, M. \& Baba, A. (2008). J. Org. Chem. 73, 9465-9468.

Noland, W. E. \& Kedrowski, B. L. (2000). Org. Lett. 2, 2109-2111.

Rigaku (2007). CrystalClear. Rigaku Corporation, Tokyo, Japan.

Sheldrick, G. M. (2008). Acta Cryst. A64, 112-122. 


\section{supporting information}

Acta Cryst. (2011). E67, o1136 [doi:10.1107/S1600536811013213]

\section{Bis(4-ethoxyphenyl) sulfoxide}

\section{Kang Meng, Cheng Wu, Jing Cao, Ping Ma and Lihong Liu}

\section{S1. Comment}

Friedel-Crafts acylation is a potent method for building acylbenzene derivatives (Edward \& Sibelle, 1963; DeHaan et al., 1979; Fillion \& Fishlock, 2005; Nishimoto et al., 2008). Thiocarbonylbenzenes may be prepared by Friedel-Crafts acylation of benzene derivatives with thiocarbonyl chloride in the presence of anhydrous aluminium chloride. Thus, in order to investigate the potentiality of the method, the title compound was prepared by Friedel-Crafts acylation of phenetol, an electron-rich benzene derivative.

In the title compound (Fig. 1) the dihedral angle between the two benzene rings ( $\mathrm{C} 3-\mathrm{C} 8$ and $\mathrm{C} 9-\mathrm{C} 14)$ is $82.7(2)^{\circ}$. The $\mathrm{S}=\mathrm{O}$ bond length is shorter than those found in previously reported arylsulfoxides (Casarini et al., 2004; Noland \& Kedrowski, 2000). The oxygen atom of the sulfoxide group is disordered over two orientations with site occupancies of 0.563 (3) and 0.437 (3) for the major and minor components, repectively. In the crystal structure, molecules are linkied by intermolecular $\mathrm{C}-\mathrm{H} \cdots \mathrm{O}$ hydrogen bonds (Table 1 ) to form chains along the $b$ axis.

\section{S2. Experimental}

A round-bottomed flask was charged with $1.19 \mathrm{~g}(10 \mathrm{mmol})$ of freshly distilled thionyl chloride, $2.44 \mathrm{~g}(20 \mathrm{mmol})$ of phenetol and $20 \mathrm{ml}$ of dried dichloromethane, and the mixture was stirred on an ice-water bath followed by addition of $2.67 \mathrm{~g}(20 \mathrm{mmol})$ of anhydrous aluminium chloride in a portionwise manner. The resulting mixture was stirred at room temperature overnight and poured into $200 \mathrm{ml}$ of ice-water. The mixture thus formed was exacted with three 50-ml portions of dichloromethane, and the combined exacts were washed with saturated brine, dried over sodium sulfate and evaporated on a rotary evaporator to afford the crude title compound. Pure title compound was obtained by column chromatography. Crystals suitable for X-ray diffraction were obtained through slow evaporation of a ethyl acetate/petroleum ether $(1: 10 v / v)$ solution.

\section{S3. Refinement}

$\mathrm{H}$ atoms were positioned geometrically and refined using a riding model, with $\mathrm{C}-\mathrm{H}=0.93-0.97 \AA$, and with $U_{\text {iso }}(\mathrm{H})=$ $1.2 U_{\mathrm{eq}}(\mathrm{C})$ or $1.5 U_{\mathrm{eq}}(\mathrm{C})$ for methyl $\mathrm{H}$ atoms. 


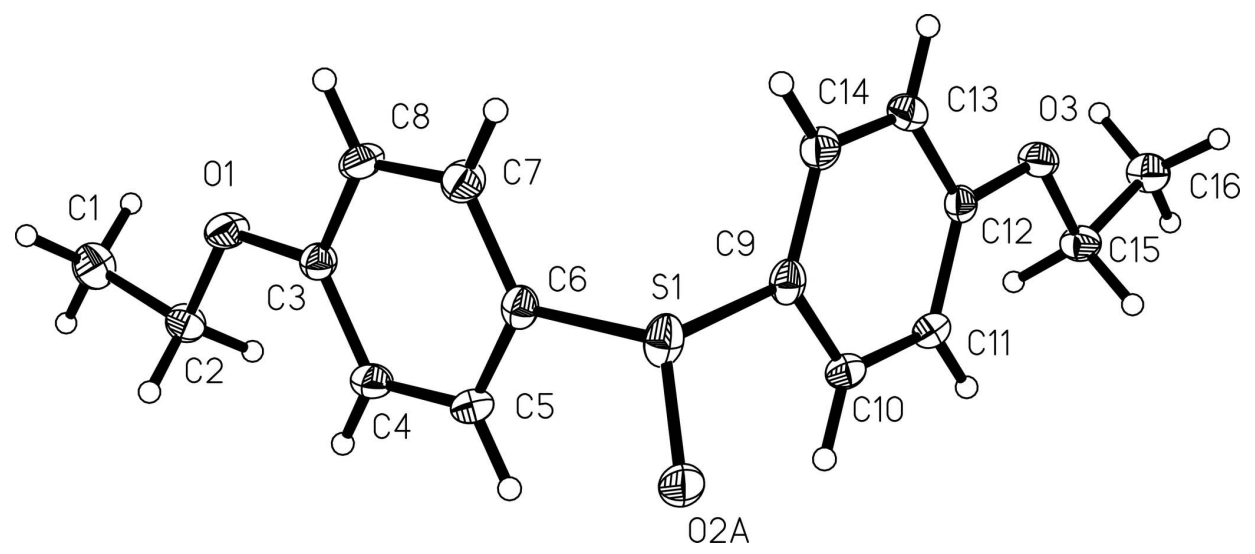

\section{Figure 1}

The molecular structure of the title compound, with $40 \%$ probability displacement ellipsoids. Only the major component of disorder is shown.

\section{Bis(4-ethoxyphenyl) sulfoxide}

\section{Crystal data}

$\mathrm{C}_{16} \mathrm{H}_{18} \mathrm{O}_{3} \mathrm{~S}$

$M_{r}=290.36$

Triclinic, $P \overline{1}$

Hall symbol: -P 1

$a=8.2052$ (16) $\AA$

$b=9.856(2) \AA$

$c=10.196(2) \AA$

$\alpha=64.71(3)^{\circ}$

$\beta=83.78(3)^{\circ}$

$\gamma=82.88(3)^{\circ}$

$V=738.4(3) \AA^{3}$

Data collection

Rigaku Saturn CCD area-detector diffractometer

Radiation source: rotating anode

Confocal monochromator

Detector resolution: 7.31 pixels $\mathrm{mm}^{-1}$

$\omega$ and $\varphi$ scans

Absorption correction: multi-scan

(CrystalClear; Rigaku, 2007)

$T_{\min }=0.957, T_{\max }=0.974$

\section{Refinement}

Refinement on $F^{2}$

Least-squares matrix: full

$R\left[F^{2}>2 \sigma\left(F^{2}\right)\right]=0.034$

$w R\left(F^{2}\right)=0.095$

$S=1.06$

3450 reflections

191 parameters

0 restraints

Primary atom site location: structure-invariant direct methods
$Z=2$

$F(000)=308$

$D_{\mathrm{x}}=1.306 \mathrm{Mg} \mathrm{m}^{-3}$

Mo $K \alpha$ radiation, $\lambda=0.71073 \AA$

Cell parameters from 2289 reflections

$\theta=2.2-27.9^{\circ}$

$\mu=0.22 \mathrm{~mm}^{-1}$

$T=113 \mathrm{~K}$

Block, colourless

$0.20 \times 0.16 \times 0.12 \mathrm{~mm}$

6624 measured reflections

3450 independent reflections

2590 reflections with $I>2 \sigma(I)$

$R_{\text {int }}=0.022$

$\theta_{\max }=27.9^{\circ}, \theta_{\min }=2.2^{\circ}$

$h=-10 \rightarrow 10$

$k=-12 \rightarrow 9$

$l=-13 \rightarrow 12$

Secondary atom site location: difference Fourier map

Hydrogen site location: inferred from neighbouring sites

$\mathrm{H}$-atom parameters constrained

$w=1 /\left[\sigma^{2}\left(F_{\mathrm{o}}^{2}\right)+(0.0422 P)^{2}+0.2002 P\right]$ where $P=\left(F_{\mathrm{o}}^{2}+2 F_{\mathrm{c}}{ }^{2}\right) / 3$

$(\Delta / \sigma)_{\max }=0.001$

$\Delta \rho_{\max }=0.26 \mathrm{e} \AA^{-3}$

$\Delta \rho_{\min }=-0.40$ e $\AA^{-3}$ 


\section{Special details}

Geometry. All e.s.d.'s (except the e.s.d. in the dihedral angle between two 1.s. planes) are estimated using the full covariance matrix. The cell e.s.d.'s are taken into account individually in the estimation of e.s.d.'s in distances, angles and torsion angles; correlations between e.s.d.'s in cell parameters are only used when they are defined by crystal symmetry. An approximate (isotropic) treatment of cell e.s.d.'s is used for estimating e.s.d.'s involving 1.s. planes.

Refinement. Refinement of $F^{2}$ against ALL reflections. The weighted $R$-factor $w R$ and goodness of fit $S$ are based on $F^{2}$, conventional $R$-factors $R$ are based on $F$, with $F$ set to zero for negative $F^{2}$. The threshold expression of $F^{2}>\sigma\left(F^{2}\right)$ is used only for calculating $R$-factors (gt) etc. and is not relevant to the choice of reflections for refinement. $R$-factors based on $F^{2}$ are statistically about twice as large as those based on $F$, and $R$ - factors based on ALL data will be even larger.

Fractional atomic coordinates and isotropic or equivalent isotropic displacement parameters $\left(\hat{A}^{2}\right)$

\begin{tabular}{|c|c|c|c|c|c|}
\hline & $x$ & $y$ & $z$ & $U_{\text {iso }} * / U_{\text {eq }}$ & Occ. $(<1)$ \\
\hline $\mathrm{S} 1$ & $-0.00304(4)$ & $0.50094(4)$ & $0.73977(4)$ & $0.02589(12)$ & \\
\hline $\mathrm{O} 1$ & $0.32089(14)$ & $-0.11895(11)$ & $0.97342(11)$ & $0.0264(2)$ & \\
\hline $\mathrm{O} 2 \mathrm{~A}$ & $-0.0884(2)$ & $0.5328(2)$ & $0.8531(2)$ & $0.0284(5)$ & $0.563(3)$ \\
\hline $\mathrm{O} 2 \mathrm{~B}$ & $-0.0815(3)$ & $0.5217(3)$ & $0.6188(3)$ & $0.0274(7)$ & $0.437(3)$ \\
\hline $\mathrm{O} 3$ & $0.60436(12)$ & $0.81887(11)$ & $0.56357(10)$ & $0.0237(2)$ & \\
\hline $\mathrm{C} 1$ & $0.4363(2)$ & $-0.35101(18)$ & $1.14478(18)$ & $0.0316(4)$ & \\
\hline H1A & 0.4553 & -0.4098 & 1.2457 & $0.047 *$ & \\
\hline H1B & 0.5396 & -0.3364 & 1.0887 & $0.047^{*}$ & \\
\hline $\mathrm{H} 1 \mathrm{C}$ & 0.3694 & -0.4028 & 1.1126 & $0.047 *$ & \\
\hline $\mathrm{C} 2$ & 0.34927 (19) & $-0.20046(16)$ & $1.12487(15)$ & $0.0248(3)$ & \\
\hline $\mathrm{H} 2 \mathrm{~A}$ & 0.2456 & -0.2138 & 1.1827 & $0.030 *$ & \\
\hline $\mathrm{H} 2 \mathrm{~B}$ & 0.4167 & -0.1460 & 1.1551 & $0.030 *$ & \\
\hline $\mathrm{C} 3$ & $0.24360(17)$ & $0.02257(15)$ & $0.92796(15)$ & 0.0205 & \\
\hline $\mathrm{C} 4$ & $0.18830(19)$ & 0.09277 (17) & $1.01871(16)$ & $0.0247(3)$ & \\
\hline H4 & 0.2018 & 0.0426 & 1.1180 & $0.030 *$ & \\
\hline $\mathrm{C} 5$ & $0.11249(18)$ & $0.23851(17)$ & $0.96065(16)$ & $0.0252(3)$ & \\
\hline H5 & 0.0752 & 0.2862 & 1.0211 & $0.030^{*}$ & \\
\hline C6 & 0.09267 (17) & $0.31234(15)$ & $0.81312(16)$ & 0.0211 & \\
\hline $\mathrm{C} 7$ & 0.14640 (19) & $0.24226(17)$ & $0.72137(16)$ & 0.0261 & \\
\hline $\mathrm{H} 7$ & 0.1315 & 0.2921 & 0.6223 & $0.031^{*}$ & \\
\hline $\mathrm{C} 8$ & $0.2223(2)$ & $0.09749(17)$ & $0.77930(16)$ & $0.0259(3)$ & \\
\hline H8 & 0.2592 & 0.0499 & 0.7188 & $0.031 *$ & \\
\hline C9 & $0.17993(17)$ & $0.59829(15)$ & $0.68666(16)$ & $0.0217(3)$ & \\
\hline $\mathrm{C} 10$ & $0.23615(18)$ & $0.64787(16)$ & $0.78032(16)$ & 0.0223 & \\
\hline H10 & 0.1784 & 0.6310 & 0.8688 & $0.027 *$ & \\
\hline $\mathrm{C} 11$ & $0.37828(17)$ & $0.72262(15)$ & $0.74289(15)$ & 0.0211 & \\
\hline H11 & 0.4161 & 0.7561 & 0.8057 & $0.025 *$ & \\
\hline C12 & $0.46333(17)$ & $0.74683(15)$ & $0.61065(15)$ & 0.0191 & \\
\hline C13 & 0.40644 (19) & $0.69756(16)$ & $0.51575(15)$ & 0.0240 & \\
\hline H13 & 0.4643 & 0.7141 & 0.4274 & $0.029 *$ & \\
\hline C14 & $0.26404(18)$ & $0.62420(16)$ & $0.55302(16)$ & 0.0240 & \\
\hline H14 & 0.2248 & 0.5924 & 0.4895 & $0.029 *$ & \\
\hline C15 & $0.67526(18)$ & $0.85931(17)$ & $0.66294(16)$ & 0.0235 & \\
\hline $\mathrm{H} 15 \mathrm{~A}$ & 0.6960 & 0.7712 & 0.7529 & $0.028 *$ & \\
\hline H15B & 0.6014 & 0.9326 & 0.6850 & $0.028 *$ & \\
\hline
\end{tabular}




\begin{tabular}{lllll} 
C16 & $0.83409(18)$ & $0.92527(18)$ & $0.58926(17)$ & $0.0278(3)$ \\
H16A & 0.8900 & 0.9472 & 0.6550 & $0.042^{*}$ \\
H16B & 0.8110 & 1.0164 & 0.5043 & $0.042^{*}$ \\
H16C & 0.9025 & 0.8543 & 0.5616 & $0.042^{*}$ \\
\hline
\end{tabular}

Atomic displacement parameters $\left(\hat{A}^{2}\right)$

\begin{tabular}{lllllll}
\hline & $U^{11}$ & $U^{22}$ & $U^{33}$ & $U^{12}$ & $U^{13}$ & $U^{23}$ \\
\hline S1 & $0.01736(17)$ & $0.02001(18)$ & $0.0400(2)$ & $0.00022(12)$ & $-0.00217(15)$ & $-0.01266(16)$ \\
O1 & $0.0373(6)$ & $0.0221(5)$ & $0.0180(5)$ & $0.0067(4)$ & $-0.0050(4)$ & $-0.0082(4)$ \\
O2A & $0.0233(10)$ & $0.0257(10)$ & $0.0363(1)$ & $-0.0013(7)$ & $0.0091(8)$ & $-0.0159(9)$ \\
O2B & $0.0276(13)$ & $0.0241(13)$ & $0.0301(14)$ & $-0.0007(10)$ & $-0.0155(10)$ & $-0.0081(11)$ \\
O3 & $0.0258(5)$ & $0.0279(6)$ & $0.0200(5)$ & $-0.0089(4)$ & $0.0021(4)$ & $-0.0116(4)$ \\
C1 & $0.0339(9)$ & $0.0257(8)$ & $0.0287(9)$ & $0.0021(6)$ & $-0.0069(7)$ & $-0.0054(7)$ \\
C2 & $0.0304(8)$ & $0.0239(7)$ & $0.0173(7)$ & $-0.0016(6)$ & $-0.0049(6)$ & $-0.0054(6)$ \\
C3 & $0.0223(7)$ & $0.0193(7)$ & $0.0199(7)$ & $-0.0006(5)$ & $-0.0017(5)$ & $-0.0082(6)$ \\
C4 & $0.0310(8)$ & $0.0258(8)$ & $0.0171(7)$ & $-0.0021(6)$ & $0.0006(6)$ & $-0.0093(6)$ \\
C5 & $0.0278(8)$ & $0.0259(8)$ & $0.0245(8)$ & $-0.0015(6)$ & $0.0049(6)$ & $-0.0150(6)$ \\
C6 & $0.0161(6)$ & $0.0194(7)$ & $0.0279(8)$ & $-0.0025(5)$ & $-0.0005(5)$ & $-0.0101(6)$ \\
C7 & $0.0338(8)$ & $0.0233(7)$ & $0.0210(7)$ & $0.0008(6)$ & $-0.0064(6)$ & $-0.0089(6)$ \\
C8 & $0.0360(8)$ & $0.0239(7)$ & $0.0201(7)$ & $0.0036(6)$ & $-0.0034(6)$ & $-0.0127(6)$ \\
C9 & $0.0189(7)$ & $0.0163(6)$ & $0.0284(8)$ & $0.0012(5)$ & $-0.0031(6)$ & $-0.0081(6)$ \\
C10 & $0.0217(7)$ & $0.0224(7)$ & $0.0240(7)$ & $0.0001(5)$ & $0.0027(6)$ & $-0.0124(6)$ \\
C11 & $0.0229(7)$ & $0.0216(7)$ & $0.0218(7)$ & $-0.0009(5)$ & $-0.0012(6)$ & $-0.0124(6)$ \\
C12 & $0.0212(7)$ & $0.0154(6)$ & $0.0195(7)$ & $-0.0012(5)$ & $-0.0017(5)$ & $-0.0061(5)$ \\
C13 & $0.0302(8)$ & $0.0241(7)$ & $0.0171(7)$ & $-0.0043(6)$ & $-0.0010(6)$ & $-0.0076(6)$ \\
C14 & $0.0301(8)$ & $0.0219(7)$ & $0.0213(7)$ & $-0.0029(6)$ & $-0.0066(6)$ & $-0.0090(6)$ \\
C15 & $0.0221(7)$ & $0.0291(8)$ & $0.0225(7)$ & $-0.0045(6)$ & $-0.0014(6)$ & $-0.0134(6)$ \\
C16 & $0.0231(7)$ & $0.0342(8)$ & $0.0285(8)$ & $-0.0065(6)$ & $-0.0001(6)$ & $-0.0149(7)$ \\
& & & & & & \\
\hline
\end{tabular}

Geometric parameters $\left(A,{ }^{\circ}\right)$

\begin{tabular}{llll}
\hline $\mathrm{S} 1-\mathrm{O} 2 \mathrm{~B}$ & $1.379(2)$ & $\mathrm{C} 6-\mathrm{C} 7$ & $1.391(2)$ \\
$\mathrm{S} 1-\mathrm{O} 2 \mathrm{~A}$ & $1.4156(18)$ & $\mathrm{C} 7-\mathrm{C} 8$ & $1.383(2)$ \\
$\mathrm{S} 1-\mathrm{C} 9$ & $1.7902(15)$ & $\mathrm{C} 7-\mathrm{H} 7$ & 0.9300 \\
$\mathrm{~S} 1-\mathrm{C} 6$ & $1.7913(16)$ & $\mathrm{C} 8-\mathrm{H} 8$ & 0.9300 \\
$\mathrm{O} 1-\mathrm{C} 3$ & $1.3619(17)$ & $\mathrm{C} 9-\mathrm{C} 10$ & $1.383(2)$ \\
$\mathrm{O} 1-\mathrm{C} 2$ & $1.4338(17)$ & $\mathrm{C} 9-\mathrm{C} 14$ & $1.393(2)$ \\
$\mathrm{O} 3-\mathrm{C} 12$ & $1.3659(17)$ & $\mathrm{C} 10-\mathrm{C} 11$ & $1.3873(19)$ \\
$\mathrm{O} 3-\mathrm{C} 15$ & $1.4331(16)$ & $\mathrm{C} 10-\mathrm{H} 10$ & 0.9300 \\
$\mathrm{C} 1-\mathrm{C} 2$ & $1.505(2)$ & $\mathrm{C} 11-\mathrm{C} 12$ & $1.387(2)$ \\
$\mathrm{C} 1-\mathrm{H} 1 \mathrm{~A}$ & 0.9600 & $\mathrm{C} 11-\mathrm{H} 11$ & 0.9300 \\
$\mathrm{C} 1-\mathrm{H} 1 \mathrm{~B}$ & 0.9600 & $\mathrm{C} 12-\mathrm{C} 13$ & $1.3949(19)$ \\
$\mathrm{C} 1-\mathrm{H} 1 \mathrm{C}$ & 0.9600 & $\mathrm{C} 13-\mathrm{C} 14$ & $1.382(2)$ \\
$\mathrm{C} 2-\mathrm{H} 2 \mathrm{~A}$ & 0.9700 & $\mathrm{C} 13-\mathrm{H} 13$ & 0.9300 \\
$\mathrm{C} 2-\mathrm{H} 2 \mathrm{~B}$ & 0.9700 & $\mathrm{C} 14-\mathrm{H} 14$ & 0.9300 \\
$\mathrm{C} 3-\mathrm{C} 4$ & $1.385(2)$ & $\mathrm{C} 15-\mathrm{C} 16$ & $1.505(2)$ \\
$\mathrm{C} 3-\mathrm{C} 8$ & $1.394(2)$ & $\mathrm{C} 15-\mathrm{H} 15 \mathrm{~A}$ & 0.9700
\end{tabular}




\begin{tabular}{|c|c|c|c|}
\hline $\mathrm{C} 4-\mathrm{C} 5$ & $1.390(2)$ & C15-H15B & 0.9700 \\
\hline $\mathrm{C} 4-\mathrm{H} 4$ & 0.9300 & $\mathrm{C} 16-\mathrm{H} 16 \mathrm{~A}$ & 0.9600 \\
\hline $\mathrm{C} 5-\mathrm{C} 6$ & $1.381(2)$ & C16-H16B & 0.9600 \\
\hline $\mathrm{C} 5-\mathrm{H} 5$ & 0.9300 & $\mathrm{C} 16-\mathrm{H} 16 \mathrm{C}$ & 0.9600 \\
\hline $\mathrm{O} 2 \mathrm{~B}-\mathrm{S} 1-\mathrm{O} 2 \mathrm{~A}$ & $120.99(14)$ & $\mathrm{C} 6-\mathrm{C} 7-\mathrm{H} 7$ & 120.4 \\
\hline $\mathrm{O} 2 \mathrm{~B}-\mathrm{S} 1-\mathrm{C} 9$ & $110.29(12)$ & $\mathrm{C} 7-\mathrm{C} 8-\mathrm{C} 3$ & $120.34(13)$ \\
\hline $\mathrm{O} 2 \mathrm{~A}-\mathrm{S} 1-\mathrm{C} 9$ & $107.71(9)$ & $\mathrm{C} 7-\mathrm{C} 8-\mathrm{H} 8$ & 119.8 \\
\hline $\mathrm{O} 2 \mathrm{~B}-\mathrm{S} 1-\mathrm{C} 6$ & $107.72(11)$ & $\mathrm{C} 3-\mathrm{C} 8-\mathrm{H} 8$ & 119.8 \\
\hline $\mathrm{O} 2 \mathrm{~A}-\mathrm{S} 1-\mathrm{C} 6$ & $109.65(10)$ & $\mathrm{C} 10-\mathrm{C} 9-\mathrm{C} 14$ & $120.52(13)$ \\
\hline $\mathrm{C} 9-\mathrm{S} 1-\mathrm{C} 6$ & 98.07 (7) & $\mathrm{C} 10-\mathrm{C} 9-\mathrm{S} 1$ & $118.90(11)$ \\
\hline $\mathrm{C} 3-\mathrm{O} 1-\mathrm{C} 2$ & $118.08(11)$ & $\mathrm{C} 14-\mathrm{C} 9-\mathrm{S} 1$ & $120.58(11)$ \\
\hline $\mathrm{C} 12-\mathrm{O} 3-\mathrm{C} 15$ & $117.39(11)$ & $\mathrm{C} 9-\mathrm{C} 10-\mathrm{C} 11$ & $120.32(13)$ \\
\hline $\mathrm{C} 2-\mathrm{C} 1-\mathrm{H} 1 \mathrm{~A}$ & 109.5 & $\mathrm{C} 9-\mathrm{C} 10-\mathrm{H} 10$ & 119.8 \\
\hline $\mathrm{C} 2-\mathrm{C} 1-\mathrm{H} 1 \mathrm{~B}$ & 109.5 & $\mathrm{C} 11-\mathrm{C} 10-\mathrm{H} 10$ & 119.8 \\
\hline $\mathrm{H} 1 \mathrm{~A}-\mathrm{C} 1-\mathrm{H} 1 \mathrm{~B}$ & 109.5 & $\mathrm{C} 12-\mathrm{C} 11-\mathrm{C} 10$ & $119.24(13)$ \\
\hline $\mathrm{C} 2-\mathrm{C} 1-\mathrm{H} 1 \mathrm{C}$ & 109.5 & $\mathrm{C} 12-\mathrm{C} 11-\mathrm{H} 11$ & 120.4 \\
\hline $\mathrm{H} 1 \mathrm{~A}-\mathrm{C} 1-\mathrm{H} 1 \mathrm{C}$ & 109.5 & $\mathrm{C} 10-\mathrm{C} 11-\mathrm{H} 11$ & 120.4 \\
\hline $\mathrm{H} 1 \mathrm{~B}-\mathrm{C} 1-\mathrm{H} 1 \mathrm{C}$ & 109.5 & $\mathrm{O} 3-\mathrm{C} 12-\mathrm{C} 11$ & $123.89(12)$ \\
\hline $\mathrm{O} 1-\mathrm{C} 2-\mathrm{C} 1$ & $107.09(12)$ & $\mathrm{O} 3-\mathrm{C} 12-\mathrm{C} 13$ & $115.57(12)$ \\
\hline $\mathrm{O} 1-\mathrm{C} 2-\mathrm{H} 2 \mathrm{~A}$ & 110.3 & $\mathrm{C} 11-\mathrm{C} 12-\mathrm{C} 13$ & $120.54(13)$ \\
\hline $\mathrm{C} 1-\mathrm{C} 2-\mathrm{H} 2 \mathrm{~A}$ & 110.3 & $\mathrm{C} 14-\mathrm{C} 13-\mathrm{C} 12$ & $120.01(13)$ \\
\hline $\mathrm{O} 1-\mathrm{C} 2-\mathrm{H} 2 \mathrm{~B}$ & 110.3 & $\mathrm{C} 14-\mathrm{C} 13-\mathrm{H} 13$ & 120.0 \\
\hline $\mathrm{C} 1-\mathrm{C} 2-\mathrm{H} 2 \mathrm{~B}$ & 110.3 & $\mathrm{C} 12-\mathrm{C} 13-\mathrm{H} 13$ & 120.0 \\
\hline $\mathrm{H} 2 \mathrm{~A}-\mathrm{C} 2-\mathrm{H} 2 \mathrm{~B}$ & 108.6 & $\mathrm{C} 13-\mathrm{C} 14-\mathrm{C} 9$ & $119.37(13)$ \\
\hline $\mathrm{O} 1-\mathrm{C} 3-\mathrm{C} 4$ & $124.36(13)$ & $\mathrm{C} 13-\mathrm{C} 14-\mathrm{H} 14$ & 120.3 \\
\hline $\mathrm{O} 1-\mathrm{C} 3-\mathrm{C} 8$ & $115.56(12)$ & $\mathrm{C} 9-\mathrm{C} 14-\mathrm{H} 14$ & 120.3 \\
\hline $\mathrm{C} 4-\mathrm{C} 3-\mathrm{C} 8$ & $120.08(13)$ & $\mathrm{O} 3-\mathrm{C} 15-\mathrm{C} 16$ & $106.61(11)$ \\
\hline $\mathrm{C} 3-\mathrm{C} 4-\mathrm{C} 5$ & $119.69(13)$ & $\mathrm{O} 3-\mathrm{C} 15-\mathrm{H} 15 \mathrm{~A}$ & 110.4 \\
\hline $\mathrm{C} 3-\mathrm{C} 4-\mathrm{H} 4$ & 120.2 & $\mathrm{C} 16-\mathrm{C} 15-\mathrm{H} 15 \mathrm{~A}$ & 110.4 \\
\hline $\mathrm{C} 5-\mathrm{C} 4-\mathrm{H} 4$ & 120.2 & $\mathrm{O} 3-\mathrm{C} 15-\mathrm{H} 15 \mathrm{~B}$ & 110.4 \\
\hline $\mathrm{C} 6-\mathrm{C} 5-\mathrm{C} 4$ & $119.88(13)$ & $\mathrm{C} 16-\mathrm{C} 15-\mathrm{H} 15 \mathrm{~B}$ & 110.4 \\
\hline $\mathrm{C} 6-\mathrm{C} 5-\mathrm{H} 5$ & 120.1 & $\mathrm{H} 15 \mathrm{~A}-\mathrm{C} 15-\mathrm{H} 15 \mathrm{~B}$ & 108.6 \\
\hline $\mathrm{C} 4-\mathrm{C} 5-\mathrm{H} 5$ & 120.1 & $\mathrm{C} 15-\mathrm{C} 16-\mathrm{H} 16 \mathrm{~A}$ & 109.5 \\
\hline $\mathrm{C} 5-\mathrm{C} 6-\mathrm{C} 7$ & $120.83(14)$ & $\mathrm{C} 15-\mathrm{C} 16-\mathrm{H} 16 \mathrm{~B}$ & 109.5 \\
\hline $\mathrm{C} 5-\mathrm{C} 6-\mathrm{S} 1$ & $119.31(11)$ & $\mathrm{H} 16 \mathrm{~A}-\mathrm{C} 16-\mathrm{H} 16 \mathrm{~B}$ & 109.5 \\
\hline $\mathrm{C} 7-\mathrm{C} 6-\mathrm{S} 1$ & $119.86(12)$ & $\mathrm{C} 15-\mathrm{C} 16-\mathrm{H} 16 \mathrm{C}$ & 109.5 \\
\hline $\mathrm{C} 8-\mathrm{C} 7-\mathrm{C} 6$ & $119.17(14)$ & $\mathrm{H} 16 \mathrm{~A}-\mathrm{C} 16-\mathrm{H} 16 \mathrm{C}$ & 109.5 \\
\hline $\mathrm{C} 8-\mathrm{C} 7-\mathrm{H} 7$ & 120.4 & $\mathrm{H} 16 \mathrm{~B}-\mathrm{C} 16-\mathrm{H} 16 \mathrm{C}$ & 109.5 \\
\hline $\mathrm{C} 3-\mathrm{O} 1-\mathrm{C} 2-\mathrm{C} 1$ & $-179.66(12)$ & $\mathrm{O} 2 \mathrm{~B}-\mathrm{S} 1-\mathrm{C} 9-\mathrm{C} 10$ & $152.14(15)$ \\
\hline $\mathrm{C} 2-\mathrm{O} 1-\mathrm{C} 3-\mathrm{C} 4$ & $-0.9(2)$ & $\mathrm{O} 2 \mathrm{~A}-\mathrm{S} 1-\mathrm{C} 9-\mathrm{C} 10$ & $18.17(15)$ \\
\hline $\mathrm{C} 2-\mathrm{O} 1-\mathrm{C} 3-\mathrm{C} 8$ & $178.78(13)$ & $\mathrm{C} 6-\mathrm{S} 1-\mathrm{C} 9-\mathrm{C} 10$ & $-95.51(12)$ \\
\hline $\mathrm{O} 1-\mathrm{C} 3-\mathrm{C} 4-\mathrm{C} 5$ & $179.25(13)$ & $\mathrm{O} 2 \mathrm{~B}-\mathrm{S} 1-\mathrm{C} 9-\mathrm{C} 14$ & $-27.63(17)$ \\
\hline $\mathrm{C} 8-\mathrm{C} 3-\mathrm{C} 4-\mathrm{C} 5$ & $-0.4(2)$ & $\mathrm{O} 2 \mathrm{~A}-\mathrm{S} 1-\mathrm{C} 9-\mathrm{C} 14$ & $-161.60(13)$ \\
\hline $\mathrm{C} 3-\mathrm{C} 4-\mathrm{C} 5-\mathrm{C} 6$ & $0.0(2)$ & $\mathrm{C} 6-\mathrm{S} 1-\mathrm{C} 9-\mathrm{C} 14$ & $84.72(13)$ \\
\hline $\mathrm{C} 4-\mathrm{C} 5-\mathrm{C} 6-\mathrm{C} 7$ & $0.6(2)$ & $\mathrm{C} 14-\mathrm{C} 9-\mathrm{C} 10-\mathrm{C} 11$ & $-0.7(2)$ \\
\hline $\mathrm{C} 4-\mathrm{C} 5-\mathrm{C} 6-\mathrm{S} 1$ & $-179.25(11)$ & $\mathrm{S} 1-\mathrm{C} 9-\mathrm{C} 10-\mathrm{C} 11$ & $179.49(11)$ \\
\hline
\end{tabular}




\begin{tabular}{ll}
$\mathrm{O} 2 \mathrm{~B}-\mathrm{S} 1-\mathrm{C} 6-\mathrm{C} 5$ & $-150.37(15)$ \\
$\mathrm{O} 2 \mathrm{~A}-\mathrm{S} 1-\mathrm{C} 6-\mathrm{C} 5$ & $-16.90(15)$ \\
$\mathrm{C} 9-\mathrm{S} 1-\mathrm{C} 6-\mathrm{C} 5$ & $95.24(13)$ \\
$\mathrm{O} 2 \mathrm{~B}-\mathrm{S} 1-\mathrm{C} 6-\mathrm{C} 7$ & $29.81(17)$ \\
$\mathrm{O} 2 \mathrm{~A}-\mathrm{S} 1-\mathrm{C} 6-\mathrm{C} 7$ & $163.29(13)$ \\
$\mathrm{C} 9-\mathrm{S} 1-\mathrm{C} 6-\mathrm{C} 7$ & $-84.58(13)$ \\
$\mathrm{C} 5-\mathrm{C} 6-\mathrm{C} 7-\mathrm{C} 8$ & $-0.8(2)$ \\
$\mathrm{S} 1-\mathrm{C} 6-\mathrm{C} 7-\mathrm{C} 8$ & $179.03(11)$ \\
$\mathrm{C} 6-\mathrm{C} 7-\mathrm{C} 8-\mathrm{C} 3$ & $0.4(2)$ \\
$\mathrm{O} 1-\mathrm{C} 3-\mathrm{C} 8-\mathrm{C} 7$ & $-179.49(13)$ \\
$\mathrm{C} 4-\mathrm{C} 3-\mathrm{C} 8-\mathrm{C} 7$ & $0.2(2)$ \\
\hline
\end{tabular}

$$
\begin{aligned}
& \mathrm{C} 9-\mathrm{C} 10-\mathrm{C} 11-\mathrm{C} 12 \\
& \mathrm{C} 15-\mathrm{O} 3-\mathrm{C} 12-\mathrm{C} 11 \\
& \mathrm{C} 15-\mathrm{O} 3-\mathrm{C} 12-\mathrm{C} 13 \\
& \mathrm{C} 10-\mathrm{C} 11-\mathrm{C} 12-\mathrm{O} 3 \\
& \mathrm{C} 10-\mathrm{C} 11-\mathrm{C} 12-\mathrm{C} 13 \\
& \mathrm{O} 3-\mathrm{C} 12-\mathrm{C} 13-\mathrm{C} 14 \\
& \mathrm{C} 11-\mathrm{C} 12-\mathrm{C} 13-\mathrm{C} 14 \\
& \mathrm{C} 12-\mathrm{C} 13-\mathrm{C} 14-\mathrm{C} 9 \\
& \mathrm{C} 10-\mathrm{C} 9-\mathrm{C} 14-\mathrm{C} 13 \\
& \mathrm{~S} 1-\mathrm{C} 9-\mathrm{C} 14-\mathrm{C} 13 \\
& \mathrm{C} 12-\mathrm{O} 3-\mathrm{C} 15-\mathrm{C} 16
\end{aligned}
$$$$
-0.1(2)
$$$$
5.76(19)
$$$$
-174.52(12)
$$$$
-179.91(13)
$$$$
0.4(2)
$$$$
-179.60(13)
$$$$
0.1(2)
$$$$
-0.9(2)
$$$$
1.2(2)
$$$$
-178.98(11)
$$

$175.76(12)$

Hydrogen-bond geometry $\left(A,{ }^{\circ}\right)$

\begin{tabular}{lllll}
\hline$D-\mathrm{H} \cdots A$ & $D-\mathrm{H}$ & $\mathrm{H} \cdots A$ & $D \cdots A$ & $D-\mathrm{H} \cdots A$ \\
\hline $\mathrm{C} 11-\mathrm{H} 11 \cdots \mathrm{O} 1^{\mathrm{i}}$ & 0.93 & 2.51 & $3.3013(18)$ & 143 \\
\hline
\end{tabular}

Symmetry code: (i) $x, y+1, z$. 